\title{
Role of bile acids in inflammatory bowel disease
}

\author{
Elisa Tiratterraa , Placido Francoc, Emanuele Porruc, Konstantinos H. Katsanos ${ }^{\mathrm{b}}$, Dimitrios K. \\ Christodoulou ${ }^{\text {, Giulia Roda }}{ }^{a}$
}

Sant'Orsola Malpighi Hospital, University of Bologna, Italy; University Hospital and Faculty of Medicine, School of Health Sciences, University of Ioannina, Greece; University of Bologna, Bologna, Italy

\begin{abstract}
Bile acids (BAs) are the end product of cholesterol catabolism. Their synthesis is regulated by the nuclear receptor farnesoid X receptor, also involved in the control of their enterohepatic circulation. Inflammatory bowel diseases (IBD), which include Crohn's disease (CD) and ulcerative colitis (UC), are multifactorial diseases characterized by diarrhea. The pathogenesis of diarrhea in IBD is still debated. The most important factor is the inflammatory process of the intestinal wall, causing alterations of solute and water absorption/secretion, deterioration of epithelial cell integrity, disruption of the intestinal microflora homeostasis, and impairment of specific transport mechanisms within the gut (including that of BAs). In this review, we summarize the current state of the art in this area and we critically evaluate the alterations of BA metabolism in patients with CD and UC.
\end{abstract}

Keywords Inflammatory bowel disease, ulcerative colitis, Crohn's disease, farnesoid X receptor, bile acids

Ann Gastroenterol 2018; 31 (3): 1-7

\section{Introduction}

Bile acids (BAs) are the end product of cholesterol catabolism; they contribute to intestinal nutrient absorption and the biliary transport of lipids, toxic metabolites, and xenobiotics [1-4]. Their synthesis is regulated by the farnesoid $\mathrm{X}$ receptor (FXR), also involved in the control of their enterohepatic circulation [2]. Inflammatory bowel disease (IBD) is a term that encompasses multifactorial diseases characterized by various symptoms, among which diarrhea represents an important target for treatment. The pathogenic role of BAs in chronic IBD has long been debated. BA malabsorption (BAM) plays a major role in diarrhea, because luminal BAs result in colonic secretion of water and electrolytes and the induction of propagated contractions.

aDepartment of Internal Medicine, Division of Gastroenterology, Sant'Orsola Malpighi Hospital, University of Bologna, Italy (Elisa

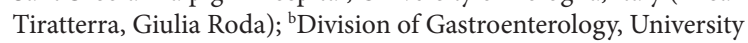
Hospital and Faculty of Medicine, School of Health Sciences, University of Ioannina, Greece (Konstantinos H. Katsanos, Dimitrios K.

Christodoulou); ${ }^{\mathrm{c}}$ Department of Chemistry G. Ciamician, University of Bologna, Bologna, Italy (Placido Franco, Emanuele Porru)

\section{Conflict of Interest: None}

Correspondence to: Giulia Roda, Department of Internal Medicine, Division of Gastroenterology, University of Bologna, Via Massarenti 9, Bologna, Italy, e-mail: giuliaroda@gmail.com

Received 24 November 2017; accepted 15 January 2018; published online 14 February 2018

DOI: https://doi.org/10.20524/aog.2018.0239
Inflammatory processes happening in the intestinal wall are the major contributing factors to IBD; nevertheless, impaired metabolism of BAs and BA-FXR interaction are involved in the pathophysiology of this disease [3]. We know that defective BA metabolism can be related to many causes, such as impaired biosynthesis from cholesterol, faulty transport of hepatocytes or enterocytes through the cellular membrane, defective transport among the physiological compartments involved in the enterohepatic circulation, or abnormal bacterial overgrowth in the large intestine [3]. Under these conditions, administration of BA sequestrants, which can reduce the colonic concentration of bile salts, leads to symptomatic improvements in diarrhea [3].

Several analytical protocols are currently used for the diagnosis of diarrhea related to BAs, including ${ }^{75}$ selenium homotaurocholic acid test retention, serum $\mathrm{C} 4$, fibroblast growth factor (FGF)-19, and fecal BA measurements. Unfortunately, these methods do not always provide consistent results and conflicting reports may be found in the literature $[5,6]$.

This review aims to summarize what is currently known about the pathogenic role of BAs and BA metabolism in IBD, providing a general overview of BA physiology and perturbations in their metabolic homeostasis, and to develop a useful tool that may be applied in clinical practice.

\section{Materials and methods}

This literature review was carried out by identifying relevant articles on the topic through an electronic search of 
the online databases on "PubMed" and "Google" from 1971 to 2017. The research was performed using a combination of terms: "bile acids", "bile salts", "malabsorption", "Crohn's disease", "ulcerative colitis", "chronic inflammatory diseases", "bile", "diarrhea", "IBD”, "cholic acid", "chenodeoxycholic acid", "lithocholic acid", "deoxycholic acid", "ursodeoxycholic acid", "primary BA", "secondary BA" and "BAs". Abstracts of all publications found by the above search strategy were screened for relevancy. Full-text articles were retrieved if available.

\section{BA metabolism}

BAs are the end product of cholesterol catabolism. Their hepatic synthesis and enterohepatic circulation ensure the homeostasis of the physiological pool. Specifically, their de novo synthesis compensates for their daily fecal loss, around $10-15 \%$ (0.5 g/day) of the total pool. The first and limiting step in BA synthesis is the hydroxylation of cholesterol in position $7 a$, catalyzed by $7 a$ cytochrome P450 (CYP7A1) to form $7 \alpha$-hydrocholesterol $[1,4]$; this takes place in the liver. The subsequent reactions include hydroxylation and sterol ring saturation, epimerization of the hydroxyl group in $3-\beta$ position, and finally conjugation with the amino acids glycine and taurine. As a result, the conjugates of the two primary BAs, cholic (CA) and chenodeoxycholic (CDCA) acids, are synthesized [7]. Indeed, before being secreted in the bile canaliculi, primary BAs are conjugated with glycine or taurine by two enzymes, the BA-CoA synthetase (BACS; also known as BA CoA ligase) and the BA-CoA amino acid $\mathrm{N}$-acetyltransferase, for the formation of amphipathic molecules used to emulsify and absorb lipids introduced with the diet [4]. The conjugation process is also required to allow an efficient $\mathrm{BA}$ secretion into bile, since the conjugates are more polar than the free form $[8,9]$. During the intestinal transit, about $95 \%$ of BAs are reabsorbed to maintain the 3-5 g of the physiological pool. Indeed, this recycling is necessary because of the limited capability of hepatocytes to produce BAs.

Conjugated BAs are absorbed only by an active mechanism. This active absorption process takes place in the lumen of the terminal ileum, where enterocytes express the apical sodiumdependent BA transporter (ASBT) or ileal BA transporter. Inside the enterocytes, BAs bind to the intestinal BA binding protein (IBABP) and finally they are discharged into the portal circulation via the organic solutes transporter (OST $\alpha / \beta)$, localized in the basolateral membrane [1].

Conjugation of BAs prevents passive absorption, allowing the conjugated BAs to be present in the intestinal lumen, where they act as a detergent for transport of insoluble lipids. Unabsorbed primary BAs are converted to secondary BAs by the enzymes of gut microbiota, mainly present in the colon. Specifically, through deconjugation and dehydroxylation of CA and CDCA, deoxycholic (DCA) and lithocholic (LCA) acids are respectively formed, while ursodeoxycholic acid (UDCA) is synthesized through the C7 epimerization of the CDCA hydroxyl group, [10]. Secondary BAs are then reabsorbed passively in the large intestine and transported via the portal circulation to the liver, where they undergo further changes.
As well as their precursors, secondary BAs are conjugated with glycine and taurine (and to a lesser extent with glucuronic or sulfuric acid), and secreted in the bile [11-13].

\section{BA-FXR-FGF-19 axis and IBD}

The FXR is a nuclear receptor belonging to the subclass of metabolic receptors $[1,2,14,15]$. BAs represent the endogenous ligands for FXR. After binding BAs, FXR dimerizes with the retinoid $\mathrm{X}$ receptor and binds as heterodimer to a sequence responsive to $\mathrm{FXR}$, regulating the transcription of several genes [16]. FXR can be activated by either free or conjugated BAs, but the stronger binding affinity is towards CDCA (EC50 = approximately $10 \mathrm{mmol} / \mathrm{L})$, with lower affinity for LCA, DCA, and CA. UDCA and hydrophilic BAs are not able to activate the receptor [17]. This enzyme is expressed in different tissues, including liver, intestine, adipose tissue, kidney, pancreas, and the vessel walls [18]. The interaction of BAs with the hepatic FXR causes an inhibition of BA synthesis; this occurs via the upregulation of the transcription of the orphan receptor small heterodimeric partner (SHP1; NR0B2). The latter inhibits the activity of CYP7A1 by inhibition of another orphan receptor, the liver receptor homolog 1 (Lrh1; NR5A2), which in turn positively regulates the CYP7A1 [19]. In an animal model, it has been shown that FXR activation induces a reduction in BA uptake by downregulation of the ASBT in enterocytes. Moreover, FXR activation promotes the excretion of BAs on the basolateral side of the cell by increasing the expression of IBABP and two membrane transporters, OST $\alpha$ and OST $\beta$, involved in the transport of BAs from the intestine to the portal circulation [20-22]. Finally, the FXR localized in enterocytes stimulates the production and secretion of FGF-19, secreted into the portal circulation and, in the liver, binds the surface FGF receptor 4 associated with the $\beta$-Klotho protein; this ligand-receptor complex activates a mitogen-activated protein kinase cascade that inhibits the activity of CYP7A1 [1,23-25].

At the intestinal level, FXR activity alleviates inflammation and preserves the integrity of the intestinal epithelial barrier by regulating the extent of the inflammatory response, maintaining the integrity and function of the intestinal barrier, preventing bacterial translocation into the intestinal tract and regulating the growth of the microbiota [26]. FXR ligands exert anti-inflammatory activities through their ability to antagonize other signaling pathways, e.g. through the interaction with other transcription factors, signal transducers and activators of transcription [27]. Several of the intestinal macrophage genes inhibited by FXR agonists are established targets for $N F-\kappa B$ genes, nuclear transcription factor genes encoding proinflammatory cytokines (tumor necrosis factor- $\alpha$, interleukins $1 \beta$ and 6 , cyclooxygenase-1, cyclooxygenase-2) and activator protein-1, which are the most important transcriptional regulators of innate and adaptive immunity in cells $[28,29]$. Gadeleta's group has shown in an animal model that FXR activation results in a decrease in epithelial permeability and proinflammatory cytokine messenger RNA expression [30]. In IBD, a dysregulation of the immune 
response is evident. As FXR and the NF- $\kappa \mathrm{B}$ are able to inhibit each other, some studies have assessed whether there is a reduction in the activation of FXR in IBD [31-33]. However, Jahnel's group has demonstrated no changes of mRNA coding for FXR in intestinal biopsies of patients with Crohn's disease (CD) and ulcerative colitis (UC) [32]. Nijmeijer's group has not demonstrated a reduced expression of FXR but a reduced activity; indeed, there is a reduced ileal expression of mRNA encoding for the receptor of target gene SHP. This indicates a decreased activity for FXR. SHP expression is reduced by $50 \%$ in patients with CD and 33\% in patients with UC [33].

\section{BA analysis for malabsorption diagnosis}

BAM can be diagnosed by analyzing BA levels in stools for their total content and for the presence of primary BAs, mainly CA. For example, through the analysis of CA and CDCA levels, it has been shown that lower postprandial peaks of CA and smaller rises in CDCA concentrations are correlated with ileal resection in the first case and ileal resections with hemicolectomy in the second [34].

Different methodologies, summarized in Table 1, are currently used for this purpose, each characterized by pros and cons. The ${ }^{14} \mathrm{C}$-glycocholate breath and stool test exploits the enzymatic deconjugation of this compound caused by an overgrown bacterial population in the small intestine. This degradation reaction release ${ }^{14} \mathrm{C}$-glycine, absorbed, metabolized in the liver and finally eliminated through breath $\mathrm{as}^{14} \mathrm{CO}_{2}$, rapidly detected. If, on the other hand, the ${ }^{14} \mathrm{C}$-glycocholate enters the large intestine, deconjugation by colonic bacteria occurs and ${ }^{14} \mathrm{C}$-glycine is excreted and measured in the feces. This test is no longer used, given the availability of easier assays that do not require radiation exposure [35].

The ${ }^{75}$ SeHCAT method is based on the use of a gamma radiation emitter semisynthetic ${ }^{75}$ selenium homotaurocholic BA, resistant to bacterial deconjugation. The assay involves the measurement of the retention of ${ }^{75} \mathrm{SeHCAT}$ in the body seven days after its oral administration. The analysis is performed by whole-body gamma counter and the amount of radioactive compound is expressed as a percentage of the administered dose. The severity of the disease is estimated at three different levels, corresponding to retention rates of $5 \%$, $10 \%$, and $15 \%[36]$.

Another method is based on the measurement of plasmatic levels of 7 A-hydroxy-4-cholesten-3-one (C4), a biomarker of BA synthesis, by liquid chromatography-tandem mass spectrometry (HPLC-MS/MS) analysis. The use of HPLCMS/MS ensures high sensitivity and specificity, making the quantification of this compound very reliable. Other advantages include the high negative predictive value (which makes this method useful for screening purposes); it is unrelated to sex, age or other potential variables and is consecutively applicable to almost all patients. The relatively low positive predictive value (about 74\%), on the other hand, necessitates further clinical evaluation [37].

The quantification of total or single BAs in feces represents another approach to BAM diagnosis. Different studies proved that higher concentrations of BAs in stools, especially CDCA and DCA, are associated with irritable bowel syndrome. Excretion of a quantity of BAs greater than $2337 \mu \mathrm{mol}$ in $48 \mathrm{~h}$ (upper limit of normal range) is used as an index of BAM. The use of instrumentation like HPLC-MS/MS, especially for the determination of single BAs, gives the method reliability but also makes it cumbersome [38].

The analysis of serum FGF-19 levels is a widely exploited assay test for BAM, considering the inverse relationship between C4 and FGF-19. The use of FGF-19 as a surrogate marker for BAM evaluation has been validated using the $\mathrm{e}^{75} \mathrm{SeHCAT}$ method as the reference standard and further validation studies are still in progress. The main advantage of this analysis procedure consists in the simplicity of using an enzymatic immunoassay [39].

Another recently reported method, less commonly used because of the complexity of the instrumentation required, is based on the analysis of the urinary biomarkers isopropanol and acetamide. The instrumentation consists of an electronic nose and a field asymmetric ion mobility mass spectrometer and this method still needs to be replicated

Table 1 Currently used methodologies for the diagnosis of bile acid (BA) malabsorption

\begin{tabular}{lll}
\hline Method & Pros & Cons \\
\hline${ }^{14}$ C-glycocholate breath/stool test & Possibility to detect small gut bacterial overgrowth & $\begin{array}{l}\text { Radiation exposure } \\
\text { Normal values can vary }\end{array}$ \\
\hline${ }^{75}$ SeHCAT method & Simplicity of the method & $\begin{array}{l}\text { Radiation exposure } \\
\text { Time-consuming analysis }\end{array}$ \\
\hline C4 measurement & No radiation and test simplicity \\
& Normal values reported in adults \\
No dependency on age/sex & Low positive predictive value \\
RA quantification by HPLC-MS & Highly reliable and specific & No radiation \\
& Possibility to measure total or single BAs & Complidation instrumentation required \\
\hline FGF-19 determination & Simplicity and rapidity & Cumbersome procedure \\
Urinary biomarker quantification & High sensitivity and specificity & Requires further validation \\
\hline
\end{tabular}

C4, 7 A-hydroxy-4-cholesten-3-one; BA, bile acid; FGF-19, fibroblast growth factor 19 
and validated $[40]$

\section{BAs and diarrhea}

BAs secreted by the small intestine play a key role in the transport and digestion of dietary lipids. Thanks to passive diffusion through the small bowel and active absorption in the terminal ileum, less than $5 \%$ of BAs get to the colon. If high BA concentrations reach the colon, secretion of fluid into the large bowel occurs, with consequent aqueous stools and erratic bowel function [41].

Three different types of BA diarrhea have been described, depending on the etiology and the original classification of BAM.

- Type 1 , or BAM, is due to ileal disease (resection or inflammation); it was described for the first time in 1967 by Hoffman, in patients with CD undergoing ileal resection, and was known as "cholera diarrhea" characterized by steatorrhea. In these cases, the ileal reabsorption capacity of BAs is reduced via an active mechanism and possibly by a loss of negative feedback based on the production of FGF-19 by enterocytes [42]. Moderate interruption of BA circulation might take place even if small ileal portions are removed, while greater interruptions occur with removal of bigger portions, beyond $100 \mathrm{~cm}$ [43].

- Type 2 is idiopathic malabsorption, caused by an inability of the enterocytes to synthetize FGF-19, which leads to a reduced negative feedback to the hepatic synthesis of BAs and consequently to their overproduction. The incidence varies between $25-32 \%$ among patients with functional diarrheal disorders [44-45].

- Type 3 is associated with: (a) cholecystectomy with a continuous flow of bile; (b) celiac disease, where the inflammatory response causes atrophy of the villi and crypt hyperplasia and damages the small intestine superficial epithelium with alterations that may extend distally over a variable distance; and (c) bacterial overgrowth syndrome, which involves impaired intraluminal transformation of dietary fats and resulting steatorrhea, caused by bacterial deconjugation of bile salts.

The pathogenesis of diarrhea in IBD is still debated. The most important factor is the inflammatory process of the intestinal wall, causing alterations of solutes and water absorption/secretion, deterioration of epithelial cell integrity, disruption of intestinal microflora homeostasis, and impairment of specific transport mechanisms within the gut (including that of BAs) $[3,46]$.

\section{UC and BAs}

Few studies have evaluated the role of BAs and diarrhea in UC. According to a study published in 1971 by Miettinen et al, diarrhea in UC patients is not caused by excessive fecal loss of bile salts, but by a decreased absorption and increased loss of water and electrolytes through the damaged colonic mucosa; the same study, therefore, assumed that a typical terminal ileum involvement of the "backwash ileitis" can cause BAM, which exacerbates the diarrhea and steatorrhea. In this specific scenario, diarrhea in UC is responsive to treatment with resins such as cholestyramine [47]. BAM is also present in patients who undergo proctocolectomy and formation of an ileo-anal pouch, a condition that generates an increased intestinal transit rate and reduces the ileal reabsorption surface; the condition is further compromised in the course of incidental pouchitis. In those patients, Hakala's group has shown a modification of the fecal BA composition with increased excretion of CA and CDCA as a consequence of reduced reabsorption, a marked reduction in the proportion of secondary BAs, DCA and LCA, normally formed by colonic bacteria, and at the same time an increased excretion of unidentified fecal BAs [48] (Table 2). However, the method used by the authors allows the quantification of the total amount of a specific BA, without discriminating between the free form and the conjugate. The alterations of the pool of BAs in UC patients not undergoing surgery are still widely debated. Gnewuch's group determined, using HPLC-MS, the level of BAs in blood samples of 161 UC patients, either with active disease or in remission. By means of this kind of analytical method, the profiling of single BAs, rather than total BAs, was performed, showing the finest variations in the BA pool in IBD. Specifically, they showed no significant alterations of the pool of BAs compared to controls (total BAs, total conjugated BAs and total glycoconjugated BAs) except for significantly lower levels of tauroconjugated BAs $(145.0 \mathrm{nmol} / \mathrm{L}$ in patients with UC vs. $344.2 \mathrm{nmol} / \mathrm{L}$ in controls) and non-conjugated BA $(471.0 \mathrm{nmol} / \mathrm{L}$ in patients with UC vs. $644.1 \mathrm{nmol} / \mathrm{L}$ in controls). However, these BAs make a minor contribution to the total BA pool and this effect is not dominant in the total BAs comparison [49] (Table 2). Besides, the authors reported that the ratio between glycoconjugate BAs and total conjugated BAs was greater compared to controls (92.7 vs. 87.4, respectively), while the ratio between tauroconjugated BAs and total conjugated BAs was smaller (7.3 vs. 12.6, respectively). Other studies demonstrated the opposite. For example, Ejderhamn's group described an increase in the serum concentration of primary BAs in young patients with UC and active disease (no significant differences in primary BA levels were recorded by Gnewuch's group) [5-49] (Table 2), while Kostic's group described a reduction in the total BA pool in serum [6] (Table 2). However, these last results cannot be properly compared with those of Gnewuch, as they are obtained from a much smaller population of patients using a different analytical method, based on radio immunoassay. Notably, in colonic biopsies from UC patients with active disease, there has been shown to be a reduction in the synthesis of mRNAs that encode for the transporter proteins of membrane for the BAs (especially for basolateral transporter and secondarily for apical transporter) and a reduction in the enzymes responsible for detoxification processes from BAs in the epithelial cells. This downregulation is significant in pancolitis, especially during flares, but not in disease forms that affect only the left colon; at the same time, it is completely reversible during the phases of remission of the disease. However, the same study did not show any alteration in FXR [32]. 


\section{$C D$ and $B A s$}

The first description of BAM in CD dates back to 1967, described by Hofmann in patients who underwent ileal resection. BAM is frequently present in patients with $\mathrm{CD}$ and, while it is frequently underestimated, this condition may cause a worsening of diarrhea with steatorrhea. Such patients may benefit from the use of BA sequestrant resins such as cholestyramine which, however, is not recommended for the treatment of patients suffering from CD in ECCO guidelines because of the numerous gastrointestinal adverse effects. Nowadays it is recognized that the severity of the BAM depends on the extension, localization and disease activity, with the highest expression occurring in forms involving the terminal ileum, where the active reabsorption of conjugated BA is impaired, while such a disorder does not occur in CD with only colonic location $[46,50]$. In ileal enterocytes from patients with a disease flare, Jahnel's group demonstrated a reduced synthesis of mRNA encoding for apical and basal transporters of BAs and reduced synthesis of BA detoxification enzymes; these alterations are irreversible during remission and explain the persistence of diarrhea. The same study, however, did not demonstrate an alteration in FXR expression [31]. Lenicek's group used the serum levels of 7a-hydroxycholest-4en-3-one (C4) measured by HPLC-MS/MS and expression of FGF-19 by enzyme-linked immunosorbent assay as surrogate markers of BAM. They demonstrated that only patients with colonic CD had C4 serum levels not different from controls, whereas non-operated $\mathrm{CD}$ patients with an affected ileum had twofold higher serum C4 levels than healthy controls, while CD patients who had undergone moderate $(70 \mathrm{~cm})$ or extensive $(>70 \mathrm{~cm})$ resection of the distal ileum had 5 - or 20 -fold greater $\mathrm{C} 4$ serum levels, respectively, compared to controls. In contrast, the FGF19 , as expected, was lower in patients with BAM compared to those without, because serum levels are indirectly proportional to C4 levels (Table 3) [46].

As regards variations in the BA amount in feces associated with the disease, Meihoff's group described high fecal excretion of BAs, a rapid intestinal transit (responsible for diarrhea) and an overall reduction in the BA pool in patients with active ileal $\mathrm{CD}$ without previous bowel resection and in patients with ileal resection [51] (Table 3).

The gold standard for the diagnosis of BAM is the ${ }^{75} \mathrm{SeHCAT}$ test, not widely available; it has a high cost and many countries, including the US, have not implemented it [52]. Studies carried out for the determination of BAM in patients with CD and ileocolonic resection showed a reduction in retention of SeHCAT with an increase in the proportion of BAs lost in the feces [53]; in serum, however, the total BA pool remains stable until the ileal resection, or when the intestinal inflammation is limited to a shorter tract of intestine and the liver, despite an increased synthesis, is no longer able to compensate for the fecal loss of BAs [53]. Indeed, in these cases, there is a quantitative reduction in BAs in serum as a consequence of their fecal loss. It is worth pointing out that a change in the qualitative pool of serum BAs has also been reported. Indeed, Gnewuch et al reported the qualitative and quantitative variation in the BA pool in 197 CD patients, determined by HPLC-MS/ MS [49]. These results showed significantly lower total BA concentrations (mainly conjugated BAs) in CD patients compared to controls, while the ratio between glycoconjugates and total conjugates was higher in patients with the disease. The loss of negative feedback based on the axis BAs-FXR-FGF19-liver generates an increase in the hepatic synthesis ex novo of the primary BAs, while secondary BAs are quantitatively reduced with a reduction in the percentage of DCA and LCA and an increased percentage of UDCA [49] (Table 3).

Since the production of secondary BAs depends on the

Table 2 Bile acids and UC

\begin{tabular}{llllll}
\hline Author & Method & Sample & Increase & Decrease & No alterations \\
\hline Hakala [48] & GLC & FECES & CA, CDCA ufBA & DCA, LCA & tBA, tcBA GCBA \\
Gneuwuch [49] & HPLC-ESI-MS & BLOOD & & TCBA ncBA & \\
Ejderham [5] & RIA & BLOOD & pBA & tBA \\
Kostic [6] & NA & BLOOD & & \\
\hline
\end{tabular}

CDCA, chenodeoxycholic acid; CA, cholic acid; DCA, deoxycholic acid; GBAs, glycoconjugated bile acids; GLC, gas liquid chromatography; LC-ESI-MS, liquid chromatography coupled to electrospray ionization (tandem) mass spectrometry; LCA, lithocholic acid; NA, non-available; ncBA, non-conjugated bile acids; $p B A$, primary bile acid; RIA, radioimmunoassay; $t B A$, total bile acids; tcBA, total conjugated bile acids; TCBA, tauroconjugated bile acids; UC, ulcerative colitis; $u f B A$, unidentified fecal bile acids

Table 3 Bile acids and CD

\begin{tabular}{|c|c|c|c|c|c|}
\hline Author & Disease localization/resection & $\mathrm{C} 4$ & FGF-19 & Feces & Serum \\
\hline \multirow[t]{2}{*}{ Meihoff [51] } & Short ileocolic resection & & & $\uparrow \mathrm{BA}$ & No alterations \\
\hline & Long ileocolic resection & & & $\uparrow \mathrm{BA}$ & $\downarrow \mathrm{BA}$ \\
\hline \multirow[t]{2}{*}{ Nolan [53] } & Short ileocolic resection & $\uparrow$ & $\downarrow$ & $\uparrow \mathrm{BA}$ & No alterations \\
\hline & Long ileocolic resection & $\uparrow$ & $\downarrow$ & $\uparrow \mathrm{BA}$ & $\downarrow \mathrm{BA}$ \\
\hline Gnewuch [49] & Ileocolic resection & & & $\uparrow \mathrm{BA}$ & $\downarrow \mathrm{BA}$ \\
\hline
\end{tabular}

BA, bile acids; C4, 7a-hydroxycholest-4en-3-one; CD, Crohn's disease; FGF-19, fibroblast growth factor 
intestinal microbiota, it is believed that this change is determined by intestinal dysbiosis that occurs in IBD. There has been shown to be a reduction in the biodiversity of commensal bacteria with reduced Firmicutes, which together with the Bacteroides represent the main bacterial commensal flora, and an increase especially during the disease flares of Lactobacilli and enterobacteria [54].

\section{Concluding remarks}

IBDs are frequently characterized by chronic diarrhea that can be due to several factors, including BAM. The purpose of this review was to evaluate the current state of art concerning the role of BAs in patients suffering from IBD. As regards UC, only four studies are available in the literature and they are in disagreement and definitely not conclusive, being unable to establish a link between BA pool changes and disease. This is due to the size and heterogeneity of both the examined populations and the methodologies used for BA determination. However, only the Gnewuch study assessed a complete BA profiling using an HPLC-MS protocol in a relatively large population, reporting a decrease in unconjugated and tauroconjugated BAs. Although this study can be considered the most reliable so far, the absence of further studies confirming these findings does not allow for any conclusions to be drawn.

On the other hand, as regards $\mathrm{CD}$, the few studies available reported an increase in BA fecal excretion, for patients with either active ileal disease or ileocolonic resection. Unfortunately, all these studies focused only on the total BA excretion, which might also be associated with an increase in intestinal motility, again making this kind of evaluation inconclusive.

Based on the available studies, BAM cannot be considered a proper diagnostic tool for $\mathrm{UC}$ and $\mathrm{CD}$, as total BAs represent a poorly specific biomarker. In ileal $\mathrm{CD}$, a potential alternative could be represented by a prompted investigation of conjugated rather than total BAs. Indeed, only conjugated BAs are reabsorbed in the ileum; thus an active ileal disease may account for an altered reabsorption of these conjugates only.

Concerning UC, future perspectives might involve the monitoring of specific BA metabolites produced by gut microbiota that have never been deeply investigated. Indeed, an altered microbial population residing in the intestine in disease states could be responsible for a qualitative and quantitative variation of BA metabolites, implying the possibility of discovering new biomarkers that could be useful as a diagnostic tool.

In conclusion, larger insights exploiting new analytical approaches are nowadays needed to expand the current knowledge on this area, with the final goal of understanding the pathogenic mechanisms underlying IBD and improving diagnostic tools and therapies currently available for the treatment of these diseases.

\section{References}

1. Gadaleta RM, van Mil SW, Oldenburg B, Siersema PD, Klomp LW, van Erpecum KJ. Bile acids and their nuclear receptor FXR:
Relevance for hepatobiliary and gastrointestinal disease. Biochim Biophys Acta 2010;1801:683-692.

2. Parks DJ, Blanchard SG, Bledsoe RK, et al. Bile acids: natural ligands for an orphan nuclear receptor. Science 1999;284:1365-1368.

3. Vítek L. Bile acid malabsorption in inflammatory bowel disease. Inflamm Bowel Dis 2015;21:476-483.

4. Philipp B. Bacterial degradation of bile salts. Appl Microbiol Biotechnol 2011;89:903-915.

5. Ejderhamn J, Strandvik B. Serum bile acids in relation to disease activity and intake of dietary fibers in juvenile ulcerative colitis. Digestion 1991;50:162-169.

6. Kostić N, Bozanić M, Cvetković R, Adamov A. [Lipids and total bile acids in the blood of patients with inflammatory bowel diseases]. Srp Arh Celok Lek 1990;118:43-46.

7. Goodwin B, Jones SA, Price RR, et al. A regulatory cascade of the nuclear receptors FXR, SHP-1, and LRH-1 represses bile acid biosynthesis. Mol Cell 2000;6:517-526.

8. Roda A, Pellicciari R, Gioiello A, et al. Semisynthetic bile acid FXR and TGR5 agonists: physicochemical properties, pharmacokinetics, and metabolism. J Pharmacol Exper Ther 2014;350:56-68.

9. Roda A, Aldini R, Camborata C, et al. Metabolic profile of obeticholic acid and endogenous bile acids in rats with decompensated liver cirrhosis. Clin Transl Sci 2017;10:292-301.

10. Agellon LB, Torchia EC. Intracellular transport of bile acids. Biochim Biophys Acta 2000;1486:198-209.

11. Hofmann AF, Hagey LR. Bile acids: chemistry, pathochemistry, biology, pathobiology, and therapeutics. Cell Molec Life Sci 2008;65:2461-2483.

12. Chiang JY. Bile acids: regulation of synthesis. J Lipid Res 2009;50:1955-1966.

13. Russell DW. Fifty years of advances in bile acid synthesis and metabolism. J Lipid Res 2009;50 (Suppl): S120-S125.

14. Makishima M, Okamoto AY, Repa JJ, et al. Identification of a nuclear receptor for bile acids. Science 1999;284:1362-1365.

15. Wang H, Chen J, Hollister K, Sowers LC, Forman BM. Endogenous bile acids are ligands for the nuclear receptor FXR/BAR. Mol Cell 1999,3:543-553.

16. Zhang Y, Edwards PA. FXR signaling in metabolic disease. FEBS Lett 2008;582:10-18.

17. Li Y, Jadhav K, Zhang Y. Bile acid receptors in non-alcoholic fatty liver disease. Biochem Pharmacol 2013;86:1517-1524.

18. Huber RM, Murphy K, Miao B, et al. Generation of multiple farnesoid-X-receptor isoforms through the use of alternative promoters. Gene 2002;290:35-43.

19. Chen F, Ma L, Dawson PA, et al. Liver receptor homologue-1 mediates species- and cell line-specific bile acid-dependent negative feedback regulation of the apical sodium-dependent bile acid transporter. J Biol Chem 2003;278:19909-19916.

20. Grober J, Zaghini I, Fujii H, et al. Identification of a bile acidresponsive element in the human ileal bile acid-binding protein gene. Involvement of the farnesoid X receptor/9-cis-retinoic acid receptor heterodimer. J Biol Chem 1999;274:29749-29754.

21. Lee H, Zhang Y, Lee FY, Nelson SF, Gonzalez FJ, Edwards PA. FXR regulates organic solute transporters alpha and beta in the adrenal gland, kidney, and intestine. J Lipid Res 2006;47:201-214.

22. Holt JA, Luo G, Billin AN, et al. Definition of a novel growth factor-dependent signal cascade for the suppression of bile acid biosynthesis. Genes Dev 2003;17:1581-1591.

23. Inagaki T, Choi M, Moschetta A, et al. Fibroblast growth factor 15 functions as an enterohepatic signal to regulate bile acid homeostasis. Cell Metab 2005;2:217-225.

24. Xie MH, Holcomb I, Deuel B, et al. FGF-19, a novel fibroblast growth factor with unique specificity for FGFR4. Cytokine 1999;11:729-735.

25. Fiorucci S, Cipriani S, Mencarelli A, Renga B, Distrutti E, Baldelli F. Counter-regulatory role of bile acid activated receptors in 
immunity and inflammation. Curr Mol Med 2010;10:579-595.

26. Kong B, Wang L, Chiang JY, Zhang Y, Klaassen CD, Guo GL. Mechanism of tissue-specific farnesoid $\mathrm{X}$ receptor in suppressing the expression of genes in bile-acid synthesis in mice. Hepatology 2012;56:1034-1043.

27. Wagner EF, Eferl R. Fos/AP-1 proteins in bone and the immune system. Immunol Rev 2005;208:126-140.

28. Ding L, Yang L, Wang Z, Huang W. Bile acid nuclear receptor FXR and digestive system diseases. Acta Pharm Sin B 2015;5:135-144.

29. Gadaleta RM, van Erpecum KJ, Oldenburg B, et al. Farnesoid $\mathrm{X}$ receptor activation inhibits inflammation and preserves the intestinal barrier in inflammatory bowel disease. Gut 2011;60:463-472.

30. Gadaleta RM, Oldenburg B, Willemsen EC, et al. Activation of bile salt nuclear receptor FXR is repressed by pro-inflammatory cytokines activating NF- $\mathrm{kB}$ signaling in the intestine. Biochim Biophys Acta 2011;1812:851-858.

31. Jahnel J, Fickert P, Hauer AC, Högenauer C, Avian A, Trauner M. Inflammatory bowel disease alters intestinal bile acid transporter expression. Drug Metab Dispos 2014;42:1423-1431.

32. Nijmeijer RM, Gadaleta RM, van Mil SW, et al. Farnesoid X receptor (FXR) activation and FXR genetic variation in inflammatory bowel disease. PLoS One 2011;6:e23745.

33. Camilleri M. Bile acid diarrhea: prevalence, pathogenesis, and therapy. Gut Liver 2015;9:332-339.

34. Aldini R, Roda A, Festi D, et al. Diagnostic value of serum primary bile acids in detecting bile acid malabsorption. Gut 1982;23:829-834.

35. Fromm H, Hofmann AF. Breath test for altered bile-acid metabolism. Lancet 1971;2:621-625.

36. Williams AJ, Merrick MV, Eastwood MA. Idiopathic bile acid malabsorption - a review of clinical presentation, diagnosis, and response to treatment. Gut 1991;32:1004-1006.

37. Camilleri M, Nadeau A, Tremaine WJ, et al. Measurement of serum 7alpha-hydroxy-4-cholesten-3-one (or 7alphaC4), a surrogate test for bile acid malabsorption in health, ileal disease and irritable bowel syndrome using liquid chromatography-tandem mass spectrometry. Neurogastroenterol Motil 2009;21:734-e43.

38. Camilleri M, Busciglio I, Acosta A, et al. Effect of increased bile acid synthesis or faecal excretion in irritable bowel syndromediarrhea. Am J Gastroenterol 2014;109:1621-1630.

39. Walters JR, Pattni SS. Managing bile acid diarrhoea. Therap $A d v$ Gastroenterol 2010;3:349-357.

40. Covington JA, Westenbrink EW, Ouaret N, et al. Application of a novel tool for diagnosing bile acid diarrhoea. Sensors (Basel)
2013;13:11899-11912.

41. Fromm H, Malavolti M. Bile acid-induced diarrhea. Clin Gastroenterolol 1986;15:567-582.

42. Hofmann AF, Poley JR. Role of bile acid malabsorption in pathogenesis of diarrhea and steatorrhea in patients with ileal resection. I. Response to cholestyramine or replacement of dietary long chain triglyceride by medium chain triglyceride. Gastroenterology 1972;62:918-934.

43. Alrefai WA, Gill RK. Bile acid transporters: structure, function, regulation and pathophysiological implications. Pharm Res 2007;24:1803-1823.

44. Hofmann AF. Chronic diarrhea caused by idiopathic bile acid malabsorption: an explanation at last. Exp Rev Gastroenterol Hepatol 2009;3:461-464.

45. Keely SJ, Walters JR. The farnesoid X receptor: good for BAD. Cell Mol Gastroenterol Hepatol 2016;2:725-732.

46. Lenicek M, Duricova D, Komarek V, et al. Bile acid malabsorption in inflammatory bowel disease: assessment by serum markers. Inflamm Bowel Dis 2011;17:1322-1327.

47. Miettinen TA. The role of bile salts in diarrhoea of patients with ulcerative colitis. Gut 1971;12:632-635.

48. Hakala K, Vuoristo M, Luukkonen P, Järvinen HJ, Miettinen TA. Impaired absorption of cholesterol and bile acids in patients with an ileoanal anastomosis. Gut 1997;41:771-777.

49. Gnewuch C, Liebisch G, Langmann T, et al. Serum bile acid profiling reflects enterohepatic detoxification state and intestinal barrier function in inflammatory bowel disease. World J Gastroenterol 2009;15:3134-3141.

50. Scaldaferri F, Pizzoferrato M, Ponziani FR, Gasbarrini G, Gasbarrini A. Use and indications of cholestyramine and bile acid sequestrants. Intern Emerg Med 2013;8:205-210.

51. Meihoff WE, Kern F Jr. Bile salt malabsorption in regional ileitis, ileal resection and mannitol-induced diarrhea. J Clin Invest 1968;47:261-267.

52. Mena Bares LM, Carmona Asenjo E, García Sánchez MV, et al. 75SeHCAT scan in bile acid malabsorption in chronic diarrhoea. Rev Esp Med Nucl Imagen Mol 2017;36:37-47.

53. Nolan JD, Johnston IM, Walters JR. Altered enterohepatic circulation of bile acids in Crohn's disease and their clinical significance: a new perspective. Expert Rev Gastroenterol Hepatol 2013;7:49-56.

54. Duboc H, Rajca S, Rainteau D, et al. Connecting dysbiosis, bileacid dysmetabolism and gut inflammation in inflammatory bowel diseases. Gut 2013;62:531-539. 